\title{
MODELADO Y SIMULACIÓN DE DISPOSITIVOS ELECTROTÉRMICOS
}

\section{Marco A. Ramírez Salinas, Luis Alfonso Villa Vargas, Héctor Báez Medina, M. en C. Cristóbal Ramírez Lazo}

Centro de Investigación en Computación, Instituto Politécnico Nacional, México. mars@cic.ipn.mx,1villa@cic.ipn.mx,hbaez@cic.ipn.mx, ralc_@hotmail.com https://doi.org/10.3926/oms.401.3.2

Ramírez Salinas, M. A., Villa Vargas, L. A., Báez Medina, H., \& Ramírez Lazo, M. en C. (2020). Modelado y simulación de dispositivos electrotérmicos. En E. San Martin Martinez, M. A. Ramírez Salinas (Eds.). Avances de investigación en Nanociencias, Micro y Nanotecnologías. Barcelona, España: OmniaScience. 85-95. 


\section{Resumen}

Los dispositivos termoeléctricos (TED), son aquellos que tienen la capacidad de generar corriente eléctrica a partir de un gradiente de temperatura, este efecto es conocido como Seebeck (1821, Tomas Johann Seebeck), en sentido contrario los dispositivos electrotérmicos son aquellos que tienen la capacidad de generar un gradiente de temperatura a partir de un flujo de corriente eléctrica, conocido como efecto Peltier (1834, Jean Peltier). Típicamente un dispositivo electrotérmico de estado sólido está compuesto de dos elementos estructurales, construidos en los últimos años con Teluro de Bismuto $\left(\mathrm{Bi}_{2} \mathrm{Te}_{3}\right)$ por su capacidad para manejar diferentes densidades de electrones libres, con el cual se han creado materiales semiconductores tipo-P por deficiencia de electrones y tipo-N por exceso de electrones, estas estructuras son conectadas eléctricamente en serie con material de cobre $(\mathrm{Cu})$ y térmicamente en paralelo por su interposición entre dos placas o sustratos aislantes de material cerámico o de Alúmina (óxido de aluminio, $\mathrm{Al}_{2} \mathrm{O}_{3}$ ), de tal forma que se exponen las interconexiones de cobre a una cara fría y otra cara caliente. En este trabajo se presenta el proceso de diseño de un dispositivo de estado sólido, su modelación y la simulación multifísica que nos ayuda a comprender este fenómeno y sus efectos.

Palabras clave: Efecto Peltier; Efecto Seebeck; Efecto Thomson; Simulación Multifísica. 


\section{Introducción}

La simulación multifísica es muy útil cuando se desea alcanzar el desempeño más alto posible ya sea de un material, un dispositivo o un sistema completo, es mucho más fácil probar y verificar su comportamiento de forma virtual, corrigiendo y mejorando un modelo y de esta forma habilitar las capacidades para la innovación.

El modelado de los dispositivos electrotérmicos requiere que por lo menos dos procesos físicos sean tomados en cuenta: Transferencia de calor por conducción en solidos y Flujo de corriente eléctrica para modelar dos o quizá los tres efectos conocidos que se dan al mismo tiempo Peltier-Seebeck-Thomson.

\subsection{Modelado Matemático}

El fenómeno físico conocido como efecto Peltier se puede explicar por el flujo de calor $J_{q}$ que acompaña al flujo de corriente eléctrica $J_{e}$ que pasa por un medio homogéneo unidimensional, y puede expresarse con el siguiente modelo:

$$
\left[\begin{array}{l}
J_{e} \\
J_{q}
\end{array}\right]=\left[\begin{array}{cc}
L_{11} & L_{21} \\
L_{12} & L_{22}
\end{array}\right]\left[\begin{array}{c}
-\frac{\Phi}{d x} \\
-\frac{T}{d x}
\end{array}\right]
$$

La matriz (L) es conocida como matriz de transporte termoeléctrico, los coeficientes $L_{i j}$ generalizan las propiedades físicas del material $(\sigma$ conductividad eléctrica, K conductividad térmica, $D$ difusión, $\rho$ resistividad eléctrica, $\Phi$ fuerza electromotriz y $T$ temperatura, y los efectos correlacionados $S$ Seebeck, $\Pi$ Peltier y $\tau$ Thomson). Aunque estos no son medibles directamente, se pueden extraer bajo algunas consideraciones. Los signos negativos se introducen para representar propiamente el comportamiento fenomenológico reportado para el flujo de calor (ley de Fourier) y flujo eléctrico (ley de ohm).

El flujo de corriente en un medio homogéneo esta dado por la ecuación siguiente:

$$
J_{e}=L_{11}\left(-\frac{\Phi}{d x}\right)+L_{12}\left(-\frac{T}{d x}\right)
$$

Para explicar el modelo de los fenómenos físicos que se describen de la ecuación (1), se harán las consideraciones siguientes. 
1) Considere que el medio homogéneo (material) se mantiene a temperatura constate $T / d x=0$ y la corriente eléctrica se genera por aplicar un voltaje externo $\Phi / d x>0$, la ecuación anterior quedaría como:

$$
J_{e}=L_{11}\left(-\frac{\Phi}{d x}\right)
$$

Si se toma en cuenta la ley de Ohm:

$$
J_{e}=\sigma\left(-\frac{\Phi}{d x}\right)
$$

Se puede asumir que en estas condiciones $L_{11}=\sigma$, que es la conductividad eléctrica.

2) una segunda consideración es que el medio homogéneo (material) está a circuito abierto $J_{e}=0$, y se aplica un gradiente de temperatura para generar el efecto Seebeck, entonces de la misma ecuación se puede obtener:

$$
L_{11}\left(\frac{\Phi}{d x}\right)=L_{12}\left(\frac{T}{d x}\right)
$$

Esto indica que la diferencia de potencial generada por un gradiente de temperatura está determinada por $S(T)=L_{12} / L_{11}$

$$
\left(\frac{\Phi}{d x}\right)=\frac{L_{12}}{L_{11}}\left(\frac{T}{d x}\right)
$$

Del modelo de la Ec. 1, el flujo de calor que acompaña al flujo de corriente en un medio homogéneo (material) está dado por:

$$
J_{q}=L_{21}\left(-\frac{\Phi}{d x}\right)+L_{22}\left(-\frac{T}{d x}\right)
$$

3) Considere que el medio homogéneo (material) se mantiene a temperatura constate $T / d x=0$ y una corriente eléctrica $J_{e}$, generada por una fuente externa $\Phi /$ $d x>0$, fluye por el medio. Debido al efecto Peltier se debe observar un flujo de corriente térmica $J_{q}$ proporcional a la corriente eléctrica, entonces se puede obtener

$$
\begin{aligned}
& J_{e}=L_{11}\left(-\frac{\Phi}{d x}\right) \\
& J_{q}=L_{21}\left(-\frac{\Phi}{d x}\right)
\end{aligned}
$$

Que un gradiente de temperatura generada por la diferencia de potencial está determinado por $\Pi(T)=L_{21} / L_{11}$, la solución de las ecuaciones anteriores quedaría como: 


$$
J_{q}=\frac{L_{21}}{L_{11}} J_{e}=\Pi J_{e}
$$

El flujo de calor es proporcional al flujo de la corriente eléctrica, y la proporcionalidad constante es $\Pi(T)=L_{21} / L_{11}$, también $\Pi(T)=S(T) T$ en donde $\Pi(T)$ es el coeficiente de Peltier, $S(T)$ es el coeficiente Seebeck, $T$ es la temperatura.

De la definición del coeficiente de Seebeck se puede observar que el voltaje de Seebeck entre dos puntos de un material homogéneo no depende de la temperatura. Los coeficientes Seebeck y Peltier esta relacionados uno con el otro a través de $\Pi(T)=S(T) T$.

\subsection{Unión de medios distintos}

Cuando existe unión de dos medios (tipo-P, tipo-N), el flujo de corriente que atraviesa el medio tipo-P es distinto al del medio tipo-N, la diferencia de estos flujos produce el calentamiento o enfriamiento en la unión dependiendo de la relación que existe entre los coeficientes y la dirección de la corriente. Para los semiconductores tipo- $\mathrm{N}$ se tiene que mientras que para semiconductores tipo-P se tiene, por lo que en el contacto N-P se tiene una distribución de temperatura como se observa en la Figura 1

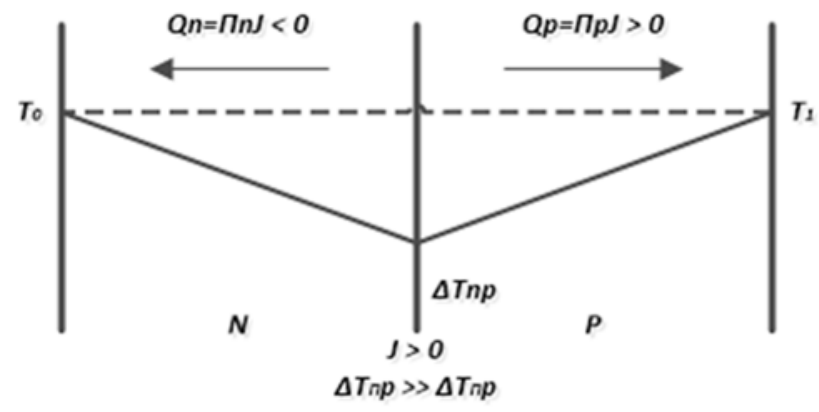

Figura 1. Distribución de temperatura en el contacto entre semiconductores tipo-P y tipo-N.

El calentamiento o enfriamiento Q que sucede en la unión P-N es:

$$
Q=\left(\Pi_{P}-\Pi_{N}\right) J_{e}
$$

Para simplificar, considere: $\Pi=\Pi_{P}-\Pi_{N}$

Así el flujo de calor que acompaña al flujo de corriente eléctrica, puede ser expresado por: 


$$
J_{q}=\Pi J_{e}-k_{e}\left(\frac{d T}{d x}\right)
$$

En donde la contribución electrónica a la conductividad térmica es:

$$
k_{e}=L_{22}\left(\frac{L_{12} L_{21}}{L_{11}}\right)
$$

Un efecto adicional puede ser agregado a la ecuación anterior, relacionado con la contribución de energía cuántica o fonónica para el flujo de calor $J_{q}$ que acompaña a un flujo de corriente $J_{e}$ a través de un medio homogéneo sólido y que se expresa en términos del calor específico por unidad de volumen a presión constante (análisis unidimensional).

$$
C p\left(\frac{d T}{d t}\right)=\frac{d}{d x}\left(k \frac{d T}{d x}\right)-\left(T \frac{d S}{d T}\right) J_{e}+\frac{J_{e}^{2}}{\sigma}
$$

Donde $C_{p}$ es el calor específico por unidad de volumen a presión constante, en un lapso de tiempo $t$. La ecuación contiene un término extra comparado con la ecuación ordinaria de conducción de calor, esto se debe al efecto Thomson, el sugiere que la distribución de temperatura (calor o frío) puede ocurrir aun en el mismo sólido, debido a la dependencia de temperatura del coeficiente Seebeck.

Cuando se produce un cambio en la energía interna del material por unidad de volumen $\Delta \mathrm{Q}$, este es proporcional al cambio de temperatura $\Delta \mathrm{T}$. Esta relación puede expresarse por:

$$
C_{p} \rho T_{t}-k T=\Delta Q
$$

Considérese: $q=-k \nabla T, \nabla \mathrm{T}=\left(\frac{\delta T_{x}}{\delta x}\right)$,

Donde $k$ es la conductividad térmica y el gradiente $\nabla$ es la derivada espacial ordinaria, $\rho$ la densidad del material,

$$
\begin{gathered}
C_{p} \nabla T=\frac{d}{d x}(k \nabla T)-\left(T \frac{d S}{d T}\right) J_{e} \frac{d T}{d x}+\frac{J_{e}^{2}}{\sigma} \\
\rho C_{p} \text { u. } \nabla T+\nabla q=Q+Q_{t e d}
\end{gathered}
$$

El coeficiente de Thomson se define por la siguiente ecuación: 


$$
\beta=\frac{\dot{q}_{c}}{J_{e} \frac{d T}{d x}}=T \frac{d S}{d T}
$$

En donde q es la velocidad de flujo de enfriamiento o calentamiento por unidad de volumen. Los tres efectos antes descritos están intrínsecamente conectados como diferentes manifestaciones del calor transportado por flujos de corriente eléctricas en un sólido cristalino. El efecto Thomson a menudo no se toma en cuenta en las simulaciones de dispositivos electrotérmicos por considerar su aportación despreciable, aunque en el análisis de materiales o nuevos materiales, si debería tomarse en cuenta.

\section{Definición del Modelo}

Una celda electrotérmica consta de dos elementos estructurales, un elemento tipo-P y un elemento tipo- $\mathrm{N}$ interconectados con cobre $(\mathrm{Cu})$. Para este análisis cada elemento es modelado con telurio de bismuto $\left(\mathrm{Bi}_{2} \mathrm{Te}_{3}\right)$ tipo-P y Tipo-N, en geometrías de tamaño $1300 \mathrm{um}$ x 1300um x 3000um, conectados con una capa de cobre de 100um de espesor. La Tabla 1, muestra las propiedades físicas de los materiales utilizados para este análisis.

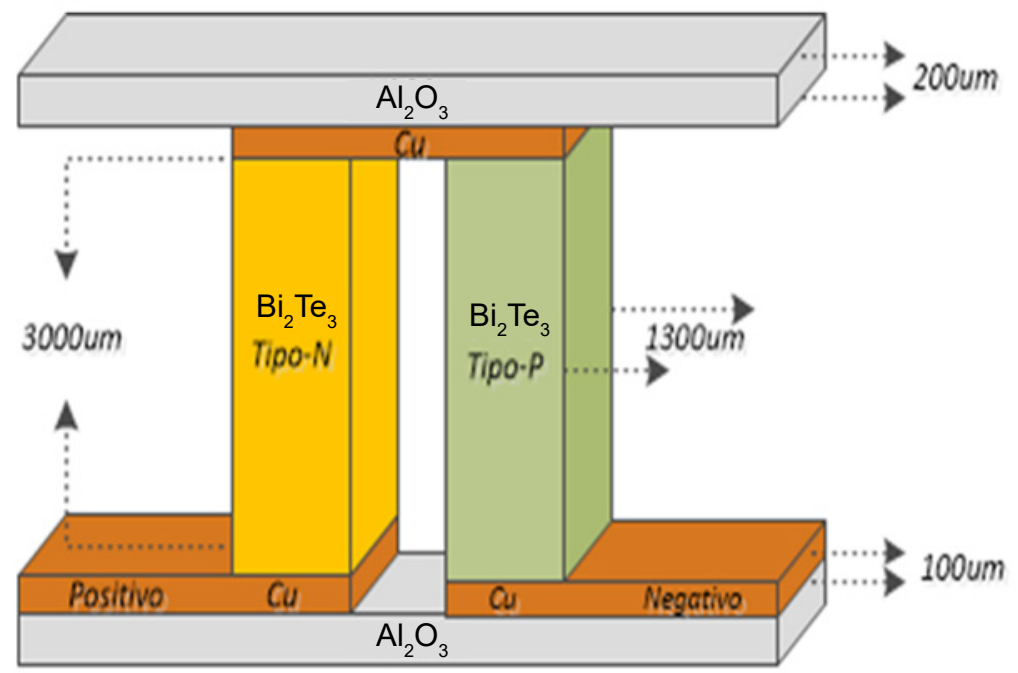

Figura 2. Celda electrotérmica unitaria. 


\section{Metodología}

COMSOL Multiphysics es una plataforma de software para simulación multifísica con una amplia biblioteca de materiales y modelos matemáticos que pueden ser combinados, para simular los fenómenos físicos y observas sus efectos lo mas cercano a la realidad. Se inicia con la herramienta Model Wizar, seleccionado el espacio de dimensión 3D, después se selecciona la física Heat Transfer y dentro de esta el modelo Termoelectric Effect, enseguida se selecciona el tipo de estudio Stationary, para calcular el campo de temperatura en equilibrio térmico. Para después crear el modelo de la Celda electrotérmica unitaria de la Figura 2.

\subsection{Materiales}

En la ventana Model Builder en Component1 se agregan los materiales de la Tabla1, utilizados en la Celda electrotérmica unitaria de la Figura 2, y posteriormente se asignan al modelo, seleccionando el material y asociándolo con la geometría correspondiente del modelo en 3D.

Tabla 1. Propiedades de los materiales.

\begin{tabular}{|c|c|c|c|}
\hline Capacidad Térmica a Presión Constante $\left(\mathrm{Bi}_{2} \mathrm{Te}_{3}\right)$ & $\mathrm{C}_{\mathrm{p}}$ & 154 & $\mathrm{~J} /(\mathrm{kg} * \mathrm{~K})$ \\
\hline Conductividad Eléctrica $\left(\mathrm{Bi}_{2} \mathrm{Te}_{3}\right)$ tipo-P & $\sigma$ & $1.1 \times 10^{5}$ & $\mathrm{~S} / \mathrm{m}$ \\
\hline Conductividad Térmica $\left(\mathrm{Bi}_{2} \mathrm{Te}_{3}\right)$ tipo-P & $\mathrm{k}$ & 1.6 & $\mathrm{~W} /(\mathrm{m} \cdot \mathrm{K})$ \\
\hline Coeficiente Seebeck $\left(\mathrm{Bi}_{2} \mathrm{Te}_{3}\right)$ tipo-P & $\mathrm{S}(\mathrm{T})$ & $2.0 \times 10^{-4}$ & $\mathrm{~V} / \mathrm{K}$ \\
\hline Coeficiente Seebeck $\left(\mathrm{Bi}_{2} \mathrm{Te}_{3}\right)$ tipo-N & $\mathrm{S}(\mathrm{T})$ & $-2.0 \times 10^{-4}$ & $\mathrm{~V} / \mathrm{K}$ \\
\hline Densidad $\left(\mathrm{Bi}_{2} \mathrm{Te}_{3}\right)$ & $\varrho$ & 7740 & $\mathrm{Kg} / \mathrm{m}^{3}$ \\
\hline Capacidad Térmica a Presión Constante $(\mathrm{Cu})$ & $\mathrm{C}_{\mathrm{p}}$ & 385 & $\mathrm{~J} /(\mathrm{kg} * \mathrm{~K})$ \\
\hline Conductividad Eléctrica $(\mathrm{Cu})$ & $\sigma$ & $5,998 \times 10^{7}$ & $\mathrm{~S} / \mathrm{m}$ \\
\hline Conductividad Térmica $(\mathrm{Cu})$ & $\mathrm{k}$ & 400 & $\mathrm{~W} /(\mathrm{m} . \mathrm{K})$ \\
\hline Coeficiente Seebeck $(\mathrm{Cu})$ & $\mathrm{S}(\mathrm{T})$ & $6.5 \times 10^{-6}$ & $\mathrm{~V} / \mathrm{K}$ \\
\hline Densidad $\left(\mathrm{Bi}_{2} \mathrm{Te}_{3}\right)$ & $\varrho$ & 8,700 & $\mathrm{Kg} / \mathrm{m}^{3}$ \\
\hline Capacidad Térmica a Presión Constante $\left(\mathrm{Al}_{2} \mathrm{O}_{3}\right)$ & $C_{p}$ & 900 & $\mathrm{~J} /(\mathrm{kg} * \mathrm{~K})$ \\
\hline Conductividad Térmica $\left(\mathrm{Al}_{2} \mathrm{O}_{3}\right)$ & $\sigma$ & 27 & $\mathrm{~W} /(\mathrm{m} \cdot \mathrm{K})$ \\
\hline Densidad $\left(\mathrm{Al}_{2} \mathrm{O}_{3}\right)$ & $\varrho$ & 3,900 & $\mathrm{Kg} / \mathrm{m}^{3}$ \\
\hline Permisividad relativa $(\mathrm{Bi} 2 \mathrm{Te} 3),(\mathrm{Cu}),\left(\mathrm{Al}_{2} \mathrm{O}_{3}\right)$ & & 1 & \\
\hline
\end{tabular}




\subsection{Fuente de Voltaje}

Es posible definir parámetros globales, seleccionando del menú Home, la opción Parameters, la idea es definir una fuente de voltaje que alimente a la celda unitaria.

\begin{tabular}{|c|c|c|c|}
\hline Name & Expresion & Value & Description \\
\hline $\mathrm{V}$ & $1[\mathrm{~V}]$ & $1 \mathrm{~V}$ & Potencial de entrada \\
\hline
\end{tabular}

\subsection{Límites de frontera}

Es importante definir los límites de frontera para la convergencia de los métodos numéricos, para este estudio en el modelo Head Transfer in Solids, en el menú Physics, se selecciona Temperature para la cara fría de Alúmina a $300^{\circ} \mathrm{K}$. Bajo la misma idea para el modelo Electric Currents, en el menú Pbysics, se selecciona Ground para el conector de cobre negativo y Electric Potential para el conector de cobre positivo, indicando $V 0$. Finalmente se puede ejecutar el cálculo, seleccionando Study1, y en seguida Compute.

\section{Resultados preliminares}

En los resultados de simulación se puede observar para la celda unitaria un gradiente de temperatura de aproximadamente $64^{\circ} \mathrm{C}$. Esto se debe principalmente a que se tiene un área térmica activa pequeña (1300um x 3500um), provocando concentración de altas temperaturas

Un arreglo de celdas unitarias como el que se muestra en la Figura 4, fue realizado para construir un dispositivo de mayor área térmica activa de $20 \times 20 \mathrm{~mm}$, en esta aproximación el gradiente de temperatura que se alcanza en toda el área activa del dispositivo es del orden de $30^{\circ} \mathrm{C}$.

\section{Conclusiones}

Las Celdas Termoeléctricas, además de aplicaciones de climatización o cosechadores de energía, en el campo de los dispositivos MEMS, pueden ser utilizados en micro reactores, actuadores a base de presión por calentamiento de gases, o para el desarrollo de cicladores térmicos programados, utilizados en equipos de 


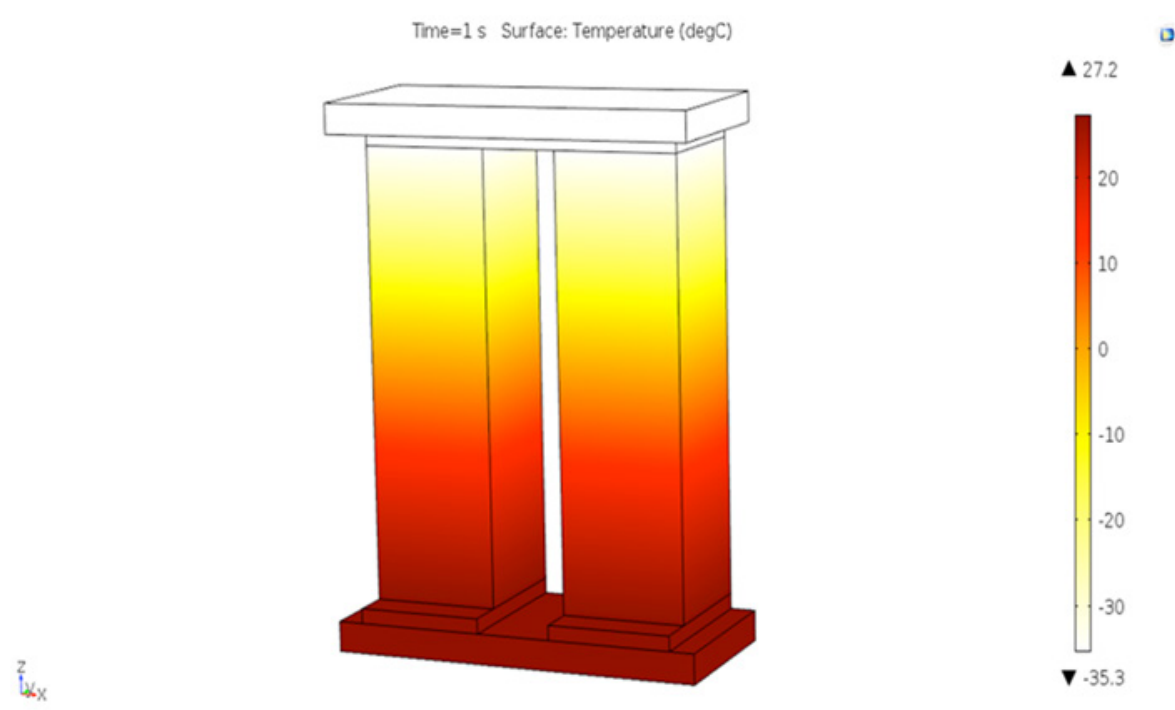

Figura 3. Simulación de la Celda electrotérmica unitaria.

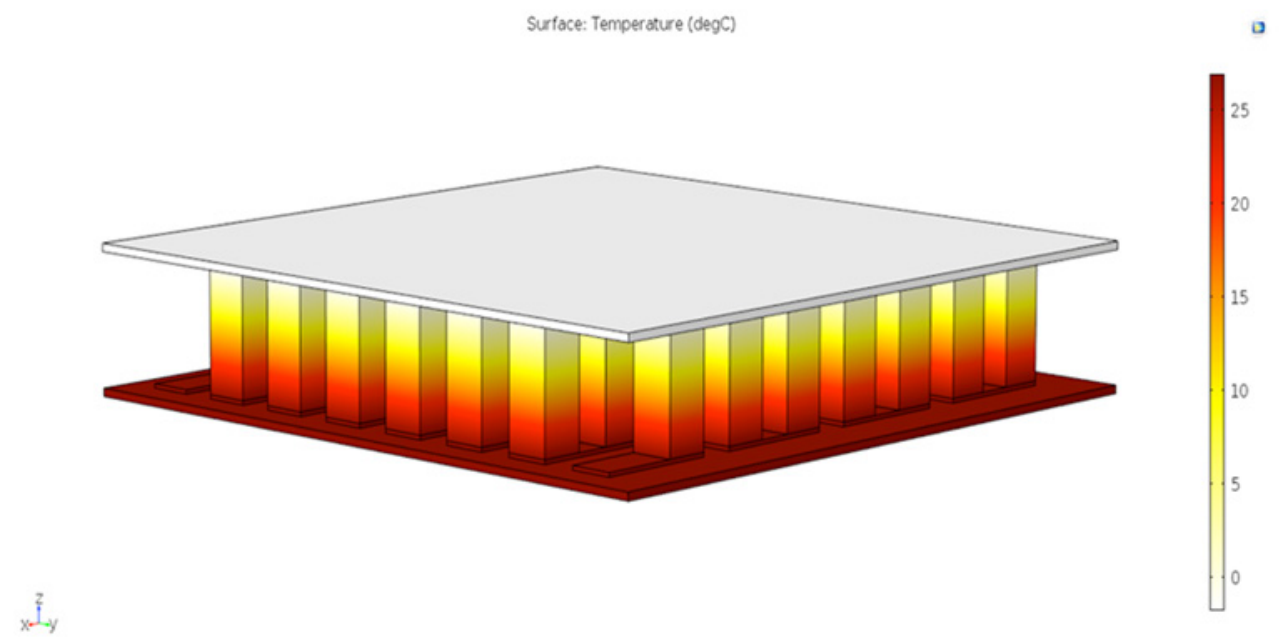

Figura 4. Arreglo de Celdas Peltier de $20 \mathrm{~mm}$ x $20 \mathrm{~mm}$.

PCR en biología molecular. La cara fría también puede ser útil para instrumentos que utilizan sensores de imágenes y que requieren enfriamiento o en su caso para análisis de muestras biológicas.

Los materiales nano estructurados con características termoeléctricas que se están desarrollando por otros grupos de la Red de Nanociencias, Micro y Nanotecnologías del IPN, buscan sintetizar buenos conductores de electricidad, pero 
malos conductores de calor para ser empleados específicamente en e cosechadores de energía, y por otro lado ser buenos conductores de calor pero malos conductores de electricidad para aplicaciones de climatización.

\section{Agradecimientos}

Agradecemos a la Secretaría de Investigación y Posgrado SIP-IPN por los apoyos otorgados a los proyectos 20160091-20170438 y 20160328- 20170444 y por el apoyo a la Red de Investigación y Posgrado en Nanociencias, Micro y Nanotecnologías y a la Red de Investigación y Posgrado en Computación.

\section{Referencias}

1. Tian, Z., Lee, S., \& Chen, G. (2014). Comprehensive Review of Heat Transfer in Thermoelectric Materials and Devices. Department of $\mathrm{Me}$ chanical Engineering, Massachusetts Institute of Technology, 17, 425-483. https://doi.org/10.1615/AnnualRevHeatTransfer.2014006932

2. Jaegle, M. (2008). Multiphysics Simulation of Thermoelectric Systems. Modeling of Peltier-Cooling and Thermoelectric Generation. Proc. COMSOL Conf. 2008 Hanover.

3. Nolas, G. S., Sharp, J., \& Goldsmid, H. J. (2001) Thermoelectrics: Basic principles and new materials developments. Springer. https://doi.org/10.1007/978-3-662-04569-5 